\title{
Relationship Between Chinese Mathematics Teachers' Knowledge and Their Professional Noticing
}

\author{
Xinrong Yang ${ }^{1} \cdot$ Gabriele Kaiser $^{2}$ • Johannes König ${ }^{3}$ • Sigrid Blömeke ${ }^{4}$
}

Received: 10 April 2019 / Accepted: 30 March 2020 / Published online: 28 April 2020

(C) The Author(s) 2020

\begin{abstract}
Although research on teachers' noticing in mathematics education has significantly increased over the last decade, little is known about the relationship between teachers' noticing and teachers' knowledge as an influential basis of their professional noticing. This paper examines this relationship based on a study involving 203 in-service Chinese mathematics teachers. The results suggest that the different components of teacher knowledge influence teacher noticing differently. Among others, the sub-facets of teachers' noticing, "interpretation and decision-making," have a stronger correlation with teachers' knowledge than the sub-facet "perception." However, due to social and cultural differences between Eastern and Western countries, the study did not identify strong connections, as expected from the results of studies carried out in Western countries. Instead, rather weak overall connections between mathematics teachers' knowledge and their noticing could be identified reflecting specific features of Chinese culture.
\end{abstract}

Keywords General pedagogical knowledge · Mathematics content knowledge · Mathematics pedagogical content knowledge $\cdot$ Teacher noticing $\cdot$ Chinese mathematics teachers

Gabriele Kaiser

gabriele.kaiser@uni-hamburg.de

Xinrong Yang

xinrongy@swu.edu.cn

Johannes König

johannes.koenig@uni-koeln.de

Sigrid Blömeke

sigrid.blomeke@cemo.uio.no

Extended author information available on the last page of the article 


\section{Introduction}

Teacher noticing has been commonly accepted as a critical component of mathematics teaching expertise, which acts as an important factor for improving the quality of teaching and students' mathematical achievements (Sherin, Jacobs, \& Philipp, 2011). Although teacher noticing is conceptualized in a variety of ways in the literature, it has been typically accepted by researchers as consisting of multiple processes or skills such as identifying, making connections, and reasoning (van Es \& Sherin, 2002), or attending, interpreting, and deciding how to respond (Jacobs, Lamb, \& Philipp, 2010) or focused under the construct of attention as raising teachers' awareness toward the students and their understanding (Mason, 1998, 2009).

Due to the importance of teacher noticing, a growing body of studies conducted by researchers in Western contexts aimed to explore factors that influence the process of its construction in order to facilitate its development. Theoretically, it has been widely accepted by Western researchers that the development of teacher noticing is "a strongly knowledge-guided process" (Stürmer, Könings, \& Seidel, 2013, p. 12), and therefore, it is "intimately tied" (Schoenfeld, 2011, p. 231) to teacher knowledge. Quite a few recent empirical studies in Western contexts indeed support the existence of relationships between teacher noticing and a single component of teacher knowledge (e.g. content knowledge or pedagogical content knowledge), although sometimes, results are partly contradictory (e.g. Dreher \& Kuntze, 2015; König et al., 2014).

However, teacher noticing is described as a "culturally shaped perception" (Ball, 2011, p. xxi), which is not considered in the abovementioned empirical studies conducted in Western culture; overall, the cultural influences to teacher noticing have been "typically neglected" (Louie, 2018, p. 61). Moreover, teacher knowledge as a mental construct is situated and developed within a specific context, and therefore, without doubt, is influenced by social and cultural characteristics from the context (Kaiser \& Blömeke, 2013). Thus, the cultural attribution of both teacher knowledge and noticing makes it meaningful and necessary to investigate to what extent the strong linkages between mathematics teacher noticing and their knowledge theoretically proposed and empirically identified in Western contexts may also be found among teachers in a different cultural context, for example, in the social and cultural context of China, an influential East Asian country with quite unique cultural traditions.

Moreover, successful noticing requires a joint functioning of a variety of different components of teacher knowledge (Dreher \& Kuntze, 2015). Therefore, it has been a challenge for the examination of the relationship between teacher noticing and single components of teacher knowledge in previous studies to provide a complete picture of the relationship between these two constructs. In addition, researchers also argued concerning the construct of teacher noticing, namely that it cannot be expected that mathematics content knowledge (MCK), mathematics pedagogical content knowledge (MPCK), and general pedagogical knowledge (GPK) are of "the same nature" (Blömeke, Busse, Kaiser, König, \& Suhl, 2016, p. 44). Therefore, an investigation of the relationship between teacher noticing and the core parts of teacher knowledge, such as GPK, MCK, and MPCK, jointly within a single study is strongly needed to see how different components of teacher knowledge relate to teacher noticing differently.

Furthermore, most current studies mainly focus on teacher knowledge differentiated along their domains, i.e. GPK, MCK, and MPCK, and noticing differentiated along a 
pedagogical or a content-related perspective. So far, few studies exist which go beyond these differentiations, evaluating at a more fine-grained level relations between subdomains of teacher knowledge and sub-facets of teacher noticing. It is expected that a deeper and more complete analysis can give insight into the complex relation between teacher knowledge and teacher noticing, especially in the light of the different strengths and weaknesses of Eastern and Western teachers in noticing from a pedagogical and a mathematics-pedagogical perspective (Yang, Kaiser, König, \& Blömeke, 2019).

In light of these reflections, the main aim of the present study is to investigate the relationship between Chinese secondary school mathematics teachers' professional knowledge (including GPK, MCK, and MPCK) and their professional noticing, and examine whether the relationship is different or similar to the structure identified within Western secondary mathematics teachers. The current study is embedded in the research program of the international comparative study Teacher Education and Development Study (TEDS-M).

\section{Literature Review}

\section{Teacher Knowledge in the Field of Mathematics}

It has been widely accepted in the respective research on teachers and teacher education that the nature of teacher knowledge is a multi-faceted construct (Ball, Thames, \& Phelps, 2008; König, Blömeke, Paine, Schmidt, \& Hsieh, 2011; Shulman, 1987). In the respective literature, researchers have proposed various frameworks to classify the components of teachers' professional knowledge after the seminal framework provided by Shulman (1987). His conceptualization of teacher knowledge has opened new perspectives of research on teacher knowledge in general and the definition of teachers' knowledge categories in particular. His differentiation mainly between content knowledge (CK), pedagogical content knowledge (PCK), and general pedagogical knowledge (GPK) is used in nearly all large-scale studies on teachers' professional competencies developed in the last decades (König et al., 2014). These three components have been considered core of teachers' professional competencies (Ball et al., 2008) and important predictors of effective mathematics instruction and students' mathematical learning (Beswick, Callingham, \& Watson, 2012; König et al., 2014).

Moreover, teacher knowledge has been accepted as a mental construct situated and developed within a specific context (Fennema \& Franke, 1992). The development process of teacher knowledge is influenced by social and cultural norms and traditions in the specific context (König et al., 2014). Therefore, the components of teacher knowledge as differentiated by Shulman (1987) for teachers in a specific context demonstrate a certain pattern of strengths and weaknesses. Clear differences of teacher knowledge were indeed identified between teachers from different contexts in previous cross-national comparative studies. For example, in TEDS-M, pre-service mathematics teachers from Eastern culture like Taiwan and Singapore were found to be particularly strong in MCK, whereas teachers from Western culture like USA and Norway were found to be particularly good in MPCK (Blömeke, Suhl, \& Döhrmann, 2013). However, in-service mathematics teachers from Taiwan were found to perform better in both MCK and MPCK than in-service mathematics teachers from Germany (Kleickmann 
et al. 2015). Similarly, differences were identified in terms of GPK among pre-service mathematics teachers from Germany, USA, and Taiwan (König et al., 2011).

\section{Mathematics Teachers' Professional Noticing}

Teacher noticing has attracted increasing research interest in the past decade, especially in the mathematics education research community in North America and Europe. In these studies, teacher professional noticing has been defined in a variety of ways (Sherin et al., 2011). However, there is currently a consensus within research on teacher noticing - that this construct can be characterized as consisting of multiple processes or components, such as attending to important classroom incidents, reasoning about or interpreting such incidents, and making decisions on how to respond (Jacobs et al., 2010; Sherin \& van Es, 2005, 2009). The identified processes or components of teacher noticing are sometimes conceptualized in a more integrated way; that is, these processes and components are described as "interrelated and cyclical" (Sherin et al., 2011, p. $5)$. By contrast, sometimes, researchers only concentrate on the analysis of a specific component, such as attending or interpreting (Thomas, 2017).

In addition, teacher professional noticing has been argued as a socially and culturally shaped construct (Louie, 2018). Therefore, investigation and analysis of teacher noticing are "inseparable from a particular context, community, and time" (Thomas, 2017, p. 510). Indeed, the latest cross-cultural comparative studies in the field found that there exists a specific pattern for teacher noticing within a specific social and cultural context. For example, it was found that German mathematics teachers demonstrated particular strengths in "perception" as a sub-facet of noticing; by contrast, Chinese teachers demonstrated strength in "analyzing and decision-making" as other sub-facets of noticing (Yang et al., 2019).

\section{Relationship Between Teacher Knowledge and Professional Noticing}

With respect to the relationship between teacher knowledge and teachers' professional noticing, there is a conceptual agreement in the Western research community that teacher noticing should be connected to their knowledge. In particular, it is widely accepted in the literature that teacher competence should be conceptualized as a continuum. Teacher noticing will then mediate between teacher disposition (e.g. knowledge and beliefs) and teacher performance in teaching practice (Kaiser, Busse, Hoth, König, \& Blömeke, 2015).

Empirical studies conducted in Western culture indeed provide evidence to support such theoretical assumption, although sometimes, findings are not always consistent. In terms of the relationship between GPK and teacher noticing, Blömeke et al. (2016) identified that compared with MCK and MPCK, teachers' GPK is more closely related to teachers' noticing. König et al. (2014) also found a (low) correlation between early career mathematics teachers' GPK and their ability to interpret classroom situations; however, no significant correlation was identified between mathematics teachers' GPK and their ability to perceive meaningful classroom situations. Such findings suggest that teachers' GPK might influence the components of their professional noticing in a differentiated way and point to the need to include a more fine-grained analysis. 
Regarding the relationship between teachers' MCK and teacher noticing, Dreher and Kuntze (2015) identified a weak but significant correlation for pre-service mathematics teachers' MCK and their noticing, but no significant correlation for in-service mathematics teachers. By contrast, König et al. (2014) found significant effects of beginning teachers' MCK on their noticing, i.e. perception and interpretation of classroom situations, and their decision-making. Similarly, Kersting, Givvin, Thompson, Santagata, and Stigler (2012) found a high correlation between in-service mathematics teachers' content knowledge and their ability to analyze classroom situations. Bruckmaier, Krauss, Blum, and Leiss (2016) also identified that MCK was significantly $(r=0.28)$ correlated with the situation-specific facets of in-service teachers. Moreover, recent studies found that MCK may influence different phases of teacher noticing differently. For example, Sánchez-Matamoros, Fernández, and Llinares (2019) found that pre-service teachers' abilities to interpret and decide are strongly connected with their content knowledge, not the ability to attend.

Concerning the relationship between teachers' MPCK and their ability to notice, Dreher and Kuntze (2015) described relatively stronger relations for in-service mathematics teachers, but not for pre-service mathematics teachers. Similarly, Bruckmaier et al. (2016) identified that in-service mathematics teachers' MPCK was significantly $(r=0.36)$ correlated with their situated reaction competency as part of their noticing competency. Dunekacke, Jenßen, Eilerts, and Blömeke (2016) even identified a rather strong relationship $(\beta=0.55)$ between pre-service preschool teachers' MPCK and their noticing competence.

\section{Own Theoretical Framework}

The current study has been carried out as East Asian extension of TEDS-Instruct (a follow-up study of TEDS-M in Germany). The theoretical framework developed in TEDS-M and TEDS-Instruct concerning the conceptualization of teacher knowledge and teacher noticing is therefore employed in this study.

For teacher knowledge, MCK has been defined as including both the basic factual knowledge of mathematics and the conceptual knowledge of structuring and organizing principles of mathematics as a discipline. That is, for mathematics teachers, a deep cognitive understanding of the mathematical content is needed to successfully accomplish their professional activities (Blömeke et al., 2016). Departing from this perspective, the present study focuses on different levels of teachers' cognitive understanding of mathematics differentiated in TEDS-M into knowing, applying, and reasoning and its correlation to noticing (Tatto et al., 2008). Different areas of mathematics, such as algebra and geometry, distinguished in TEDS-M were not considered in this study as influences of various areas of mathematics on teachers' noticing were not expected.

Similarly, in the TEDS-M study, the following sub-domains of MPCK were differentiated: (a) curricular knowledge and knowledge of planning for mathematics teaching and learning; (b) knowledge of enacting mathematics for teaching and learning (Tatto et al., 2008). The first sub-domain mainly refers to knowledge at the pre-active stage, such as to establish appropriate learning goals, see connections within the curriculum, and plan appropriate activities and methods. The second sub-domain refers to knowledge at the interactive stage, including knowledge to analyze and evaluate students' 
mathematical solutions and arguments, provide appropriate feedback, and analyze and diagnose students' questions (Tatto et al., 2008).

For GPK, in the framework of TEDS-M, four essential common parts were identified covering instructional models used across countries to describe effective teaching (König et al., 2011; König et al., 2014). The four common tasks were as follows: (a) preparing, structuring, and evaluating lessons ("structure"); (b) motivating and supporting student learning, and managing the classroom ("motivation/ classroom management"); (c) dealing with heterogeneous learning groups in the classroom ("adaptivity"); (d) assessing students ("assessment"). This part of TEDS$M$ was conducted in Germany, the USA, and Taiwan. Since the educational culture in the part of Mainland China where the present study was conducted is similar to the culture in Taiwan, these four dimensions of GPK were also adopted to assess teachers' GPK in the present study.

For the framework of teacher noticing, the various follow-up studies of the international comparative study TEDS-M, i.e. TEDS-FU, TEDS-Instruct, and TEDS-Validate, conceptualized teachers' professional noticing as a construct oriented along classroom situations, enriching the systematic disciplinary cognitive perspective of the theoretical framework of the original study TEDS-M (see Kaiser et al., 2015; Kaiser et al., 2017). Referring in addition to the work by Berliner (2001) and Jacobs et al. (2010), the theoretical framework has been further developed in the various TEDS follow-up studies by adding and defining teacher noticing as "Perception, Interpretation, and Decision-making" called PID model (Kaiser et al., 2015). The following sub-facets of noticing were distinguished: (a) perceiving particular events in an instructional setting; (b) interpreting the perceived activities in the instructional setting; (c) decision-making, either as anticipating responses to students' activities or as proposing alternative instructional strategies. This model was developed specifically to meet the need to investigate teacher situation-specific skills, which complement the knowledge-based facets of teacher competence (Kaiser et al., 2015; Kaiser et al., 2017) and go beyond other conceptions on noticing.

Overall, this extended construct on teachers' noticing covers three processes, which require teachers not only to perceive particular events and interpret them, but also to make further decisions and develop reasonable actions based on subject-related misconceptions or errors of students, or teacher's structuring of the lesson. In addition, this extended construct on teachers' noticing does not limit teachers' professional noticing to a specific aspect of teacher work, like focusing on students' thinking, students' strategies, or student reasoning (e.g. Jacobs et al., 2010; Sherin \& van Es, 2009), but includes subject-specific aspects, too, taking up reflections using the approach by Mason on teachers' awareness and attention (Mason, 1998, 2009). This extended construct comprises a broad understanding of teacher noticing, not limited to in-the-moment noticing (Stockero, Ropnow, \& Pascoe, 2017), but including all the aspects important for the quality of mathematics teaching, such as the design of mathematical teaching and learning processes, the potential of cognitive activation of students, individual learning support, and classroom management (Kaiser et al., 2015). Particularly, this model mainly differentiates teachers' professional noticing in two sub-domains: general pedagogical aspects (labeled P_PID) and mathematics instructional aspects (labeled M_PID). 
Within this framework and based on the current findings and gaps identified in the current empirical studies on the relation between teachers' professional noticing and their knowledge, the following research questions were formulated for the present study:

1. What are the relations between teacher knowledge and teacher noticing at a global level and further differentiated at a deeper level considering the three sub-facets of noticing?

2. How far can the relations between teacher knowledge and teacher noticing identified in studies with Western teachers be reconstructed within a sample of East Asian teachers?

To answer these two questions, we developed a main hypothesis and three subhypotheses:

Main hypothesis H0: Considering the cultural attribution of teacher knowledge and teacher noticing, we assume that the strength of the relationship between these two constructs in the Chinese context will be different to the relationship being theoretically proposed and empirically identified in Western culture.

\section{Sub-hypotheses:}

H1: Considering the difference of the nature of the different domains of teacher knowledge, we assume that they will relate to teacher noticing (differentiated by P_PID and M_PID) differently between Eastern and Western teachers. More specifically, we assume a stronger relationship between MPCK and their noticing compared with the relationship between teachers' MCK and their noticing for both groups and a weaker correlation between GPK and noticing for Chinese compared with Western teachers.

H2: We assume a strong relationship between GPK and P_PID on the one hand and between MPCK and M_PID on the other hand compared with other relationships, given the joint generic nature of the constructs in the first case and the joint mathematics-related nature of the constructs in the latter case. However, we expect weaker relations for GPK and P_PID for the Chinese teachers due to the lower importance of these aspects in the Chinese context.

H3: Considering the fact that teacher noticing has been widely accepted as a process with several phases, it is assumed that teacher knowledge, especially different levels of cognitive understanding of MCK and different sub-domains of GPK and MPCK, will function differently at different phases of teacher noticing. We do not have hypotheses about the direction of the differences between East Asian and Western teachers.

\section{Methodology}

\section{Research Site and Participants}

The sample in the present study consists of 203 junior secondary school mathematics teachers who were chosen from 19 junior secondary schools in a district in Chongqing, one of the four Chinese municipalities and the only one in the Western part of China. We approached a total of 227 teachers in the district, and 208 teachers attended the study, but 5 of them did not complete the whole test. In terms of its economic situation, 
Chongqing is at the middle level in Mainland China, which means that economically, it is more representative of China than the more economically developed or underdeveloped regions. Unlike the other municipalities, Chongqing has a comparatively low urbanization level with a ratio of around $50 \%$, which also makes it more representative of the current situation of China.

The 203 participating teachers were chosen from schools with different academic backgrounds (selective and non-selective schools) and school locations (rural and urban). Among them, 105 teachers (52\%) were female and 69 (34\%) taught in rural schools at the time the assessment was carried out in December 2016. Their experience in teaching junior secondary school mathematics ranged from 2 to 36 years, with an average of 13 years. A total of 25 teachers (12\%) held a master's degree and the other $178(88 \%)$ held a 4-year bachelor degree in mathematics. The 203 teachers were from the same district and were invited to attend a 2-day professional development program. The assessment was conducted in the middle of the program. The teachers were informed that their answers will be used for empirical studies on teachers' competencies. Those who did not want to be assessed did not attend the test. The 203 teachers were assigned randomly to one of five lecture rooms and completed a paper-and-pencil test within $2.5 \mathrm{~h}$ (they had a 15-min break between the knowledge test and the noticing test).

\section{Assessment Instruments and Validation}

Assessment Instruments of Teacher Knowledge and Validation Process. To assess the professional knowledge of mathematics teachers in China, the instruments developed in TEDS-FU and used in TEDS-Instruct were adapted in this study. The items used to assess teachers' GPK, MCK, and MPCK were taken from TEDS-FU and TEDSInstruct and stemmed originally from the released items of TEDS-M, where they had already been validated within an East Asian context (König et al., 2014; Tatto et al., 2008). Referring to the joint, deeply rooted cultural values on mathematics education shaped by Confucian heritage culture (CHC) similar for most East Asian regions (Leung, 2001), this part of the instruments was directly used in the present study to test teachers' knowledge in Chongqing.

As the assessment consisted of three knowledge tests lasting 60 min altogether, the number of items used in TEDS-FU and TEDS-Instruct was reduced compared with that in TEDS-M to lessen the test burden for the participants, but without lowering the reliability (Blömeke et al., 2016; König et al., 2014). As the translations of the TEDS$\mathrm{M}$ instruments in Taiwan were written in traditional Chinese, they were modified into simplified Chinese writing to meet the mathematics education situation in Chongqing. To confirm the content validity of the three scales, a Chinese expert group consisting of two professors in mathematics education and six highly experienced junior secondary school mathematics teachers evaluated the adequacy of the modifications and made slight modifications.

Twenty-two dichotomously coded items were used to assess MCK. Open response answers were required for eight items, and the other 14 items were multiple-choice items. The 22 items covered content in number, algebra, and geometry as core areas of junior secondary school mathematics. They also encompassed the cognitive domains of knowing, applying, and reasoning. A total of 24 dichotomously coded items were used to assess MPCK, with four open-constructed items and 20 multiple-choice items. The 
items covered aspects of curricular knowledge and knowledge of planning for mathematics teaching and learning (called curriculum/planning) and knowledge of enacting mathematics for teaching and learning (called enacting). To assess Chinese mathematics teachers' GPK, 22 dichotomously coded items were used and relatively equally distributed across the four sub-domains of GPK: lesson planning ("structure"), motivating student learning and managing the classroom ("motivation/classroom management"), dealing with heterogeneity ("adaptivity"), and assessing students ("assessment").

Assessment Instruments of Teachers' Professional Noticing and Validation. In TEDSFU, three video-vignettes were developed to assess German mathematics teachers' professional noticing (for details on these instruments, see Kaiser et al., 2015). The video assessment intended to examine teachers' domain-specific and general pedagogical perception, interpretation, and decision-making skills. The video-vignettes were developed using scripts that covered critical incidents of mathematics teaching and the full range of typical teaching phases during a mathematics lesson. Mathematical topics covered in the three videos included functions, volumes, and surfaces usually taught in grades 8 to 10 according to German mathematics curricula. The three video-vignettes are not episodes taken from real classroom teaching, but scripted plots with different teachers and students. Each video-vignette lasts up to around 4 min with the aim to provide an overview of the whole lesson. To help participants gain a more comprehensive understanding of the videotaped teaching, background information about the class and lessons prior to the lesson videotaped were provided.

In Fig. 1, examples of items are given to investigate teachers' noticing on both M_PID and P_PID by describing essential aspects from the perspective of mathematics education and general pedagogy, respectively.

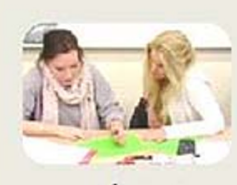

1

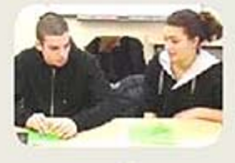

2

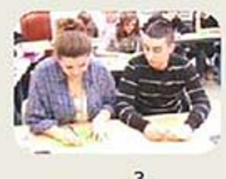

In the video-vignette the working processes of three cooperating pairs have been observed more closely. These working processes are to be examined from two perspectives: (a) mathematics education and (b) pedagogics.

(a) Mathematics education perspective In each of the three approaches the task is represented and solved mathematically in a specific way.

Please describe (in note form) the essential aspects of the approaches in a contrasting mode from a mathematics education view.

Please name - if possible - the corresponding technical terms.

(b) Pedagogics perspective Please describe (in note form) for each of the three pairs in a contrasting mode the essential aspects of the way the two students cooperated in their work.

Fig. 1 Example of a high-inferential M_PID and P_PID item referring to interpreting a classroom situation (from Kaiser et al., 2015, p. 380f) 
After watching each of the three videos, the mathematics teachers were asked to answer the items. The testing time was $60 \mathrm{~min}$ overall. The items either focused on perception, interpretation, and decision-making under a pedagogical perspective (labeled P_PID) or under the perspective of mathematics instruction (labeled M_PID). The former mainly examined teachers' general pedagogy-related noticing and the latter teachers' mathematics instruction-related noticing. Generally, the items required teachers to notice mathematics classroom teaching as a whole process, that is, items related to almost all the aspects of classroom teaching.

In total, there were 38 items (22 P_PID and 16 M_PID) based on Likert scales (four categories ranging, for example, from "fully correct" to "not correct") to assess the teachers' perception. There were 36 constructed-response items (18 P_PID and 18 M_PID) to assess the teachers' interpretation and decision abilities. An expert rating was implemented to decide which answer could be regarded as correct with respect to the rating scales. A coding manual was developed with respect to the constructed responses through a systematic analysis of related theories and literature and discussions among experienced mathematics teachers and mathematics education researchers. The coding manual was piloted before it was used in TEDS-FU and TEDS-Instruct to improve its reliability and validity. Many different approaches, like curricular analyses of the mathematical content and comprehensive expert workshops, were employed to ensure the content validity in the project (Blömeke et al., 2016).

The instruments were adapted in the present study to investigate mathematics teachers' professional noticing in China (for a detailed description of the adaptation and validation process, see Yang, Kaiser, König, \& Blömeke, 2018). The process of translation and crosscultural adaptation of the instrument of teacher noticing was carried out following the International Test Commission (2017) guidelines. In detail, the instruments were translated from German to English by a native German mathematics education master student and checked by several German mathematics educators. The translation from English into Chinese was checked by several Chinese mathematics educators in order to secure the semantic, idiomatic, experiential, and conceptual equivalence between the original German items and the Chinese translation (Hambleton, 2005).

Based on further feasibility analyses with Chinese mathematics educators and Chinese junior secondary school mathematics teachers, several items from the German instrument, closely related to German mathematics curriculum and heterogeneity or multiple cultural backgrounds of students, were excluded because they did not match the situation in Chongqing. Three Chinese junior secondary school mathematics teachers and their students retook the three videos and performed exactly as their German counterparts did.

For further validation of the instrument of teacher noticing in a Chinese context, both qualitative and quantitative methods were employed in the present study (see Yang et al., 2018 for detailed information). Besides the content validity mentioned above, "elemental validity" of the instrument was examined referring to approaches by Hill, Dean, and Goffney (2007) and Kane (2001). Cognitive interviews were conducted with eight junior secondary mathematics teachers and examined concerning the consistency and correctness of their thinking and reasoning with the answers they gave and the relation to the type of knowledge intended to measure. The overall elemental validity results showed that for almost all items, the Chinese teachers provided evidence that they understood the items without difficulty and applied the knowledge and skills that the items intended to examine other than employing test-taking strategies. 
Furthermore, construct validity was examined based on the evaluation of the psychometrical properties of the five assessment domains. Confirmatory factor analyses were performed using Mplus to validate the structure of GPK, MCK, MPCK, P_PID, and M_PID separately with the data collected from the 203 Chinese mathematics teachers. Due to the relatively small number of participants in the present study $(N=203)$, parcels of items were used, and the resulting item mean scores of each of the parcels were used as manifest indicators to produce more stable factor solutions (Little, Cunningham, Shahar, \& Widaman, 2002). When conducting structural equation modeling, Bentler and Chou (1987) recommended having at least five cases in the sample for one parameter to be estimated. Consequently, with a sample of $n=203$ cases, scaling analysis on the level of single items of the five tests was not possible. Item parcels summarized the information for the theoretically based subscales of each construct; that is, sum scores for each of the variables outlined in Table 1 were used. The reliability of the subscales was mostly acceptable, expect for a few.

At this step, the mathematical domains of number, algebra, and geometry were used as the focus of MCK. The focus of GPK, MPCK, P_PID, and M_PID is described in Table 2. Items with the same task stem within the knowledge test, and items testing a similar topic (e.g. classroom management, teacher behavior, and student errors) in the video-based part of the test were assigned to one parcel to account for potential taskspecific variance. The model fit of the five models is reported in Table 1. Various fit indices were used to evaluate the fit of each of the five models such as the ratio of chisquare values to the degrees of freedom $\left(\chi^{2} / \mathrm{df}\right)$, root mean square error of approximation (RMSEA), standardized root mean square residual (SRMR), and the comparative fit index (CFI). Based on these criteria (see Table 1), confirmatory factor analyses showed that the five models fit well or are acceptable to the data, which confirmed the structure of the five constructs GPK, MCK, MPCK, P_PID, and M_PID as separate domains for Chinese teachers' professional competencies.

\section{Scaling and Data Analysis}

The data analysis comprised the following steps. First, the constructed response items were coded according to the coding rubrics developed in the studies TEDS-M, TEDS-

Table 1 Model fit indices for the five domains assessed (Yang et al., 2018)

\begin{tabular}{llllll}
\hline Assessment & $\chi^{2 / \mathrm{df}}$ & $p$ value & CFI & RMSEA & SRMR \\
\hline GPK & 4.81 & $p<0.001$ & 0.944 & 0.038 & 0.061 \\
MCK & 2.63 & $p<0.001$ & 0.973 & 0.016 & 0.060 \\
MPCK & 4.78 & $p<0.001$ & 0.984 & 0.020 & 0.046 \\
P_PID & 4.31 & $p<0.001$ & 0.989 & 0.014 & 0.033 \\
M_PID & 2.74 & $p<0.001$ & 0.961 & 0.023 & 0.047 \\
\hline
\end{tabular}

$G P K$, general pedagogical knowledge; $M C K$, mathematics content knowledge; $M P C K$, mathematics pedagogical content knowledge; $P_{-} P I D$, pedagogical perception, interpretation and decision-making skills; $M \_P I D$, mathematical perception, interpretation and decision-making skills; $\chi^{2}$, chi-square; $d f$, degrees of freedom; CFI, comparative fit index; RMSEA, root mean square error of approximation; SRMR, standardized root mean square residual 
Table 2 Basic statistics of the study variables (reliabilities, range, mean, and standard deviation)

\begin{tabular}{lrrrrr}
\hline Variable & Number of items & $\alpha$ & Min. & Max. & M \pm SD \\
\hline GPK & 22 & & -1.68 & 1.65 & $0.01 \pm 0.61$ \\
$\quad$ Structure & 7 & 0.85 & -4.00 & 3.27 & $0.05 \pm 1.49$ \\
Motivation & 5 & 0.72 & -4.19 & 1.31 & $0.14 \pm 1.19$ \\
Adaptivity & 6 & 0.56 & -4.58 & 3.60 & $0.00 \pm 1.25$ \\
Assessment & 4 & 0.74 & -2.46 & 0.94 & $0.03 \pm 0.84$ \\
MPCK & 22 & & -2.31 & 1.81 & $0.01 \pm 0.74$ \\
Curriculum & 8 & 0.57 & -2.33 & 2.10 & $0.01 \pm 0.97$ \\
Planning and enacting & 14 & 0.85 & -2.65 & 2.53 & $0.02 \pm 0.82$ \\
MCK & 20 & & -2.07 & 3.77 & $0.00 \pm 0.95$ \\
Knowing & 6 & 0.68 & -3.10 & 0.81 & $-0.34 \pm 1.15$ \\
Applying & 8 & 0.70 & -2.35 & 1.00 & $-0.11 \pm 0.92$ \\
Reasoning & 6 & 0.66 & -3.52 & 3.82 & $0.00 \pm 1.31$ \\
P_PID & 26 & & -1.61 & 2.93 & $0.01 \pm 0.77$ \\
Perception & 20 & 0.76 & -1.95 & 2.64 & $0.01 \pm 0.89$ \\
Interpretation and decision-making & 6 & 0.81 & -3.91 & 2.89 & $0.01 \pm 1.41$ \\
M_PID & 35 & & -1.61 & 1.75 & $0.01 \pm 0.61$ \\
Perception & 18 & 0.68 & -1.54 & 1.72 & $0.00 \pm 0.63$ \\
Interpretation and decision-making & 17 & -4.08 & 2.72 & $0.00 \pm 1.09$ \\
\hline & & & & & \\
\hline
\end{tabular}

FU, and TEDS-Instruct. Two independent raters coded $25 \%$ of the questionnaire; good values of Cohen's Kappa were reached $\left(k>0.76\right.$ and $\left.K_{\text {average }}=0.84\right)$. For all the constructed response items, items with no response or an incorrect response were scored 0 , and each correct answer was scored 1 (for items with several sub-items, the sum of the correct answers was calculated). After the completion of coding, the relative item difficulties for a one-parameter (Rasch model) item response theory (IRT) model were calculated separately on the five dimensions of GPK, MCK, MPCK, P_PID, and M_PID. Items with extreme difficulty were removed for the final analysis, as they do not substantially contribute to the measurement of the construct due to weak discrimination (Bond \& Fox, 2007). The internal consistency of the remaining items in the subdimensions of the five dimensions was estimated using Cronbach's alpha reliability coefficient (see Table 2 for the descriptive results for the variables). As shown in Table 2, the reliability scores for each variable were mostly acceptable or good, except for a few subscales such as "Adaptivity" of GPK and "Curriculum" of MPCK. However, for theoretical reasons, we decided to keep these variables. These relevant methodological limitations will be reflected in the last section. After this, scale scores were created for each of the five dimensions and their sub-dimensions by applying the one-dimensional Rasch model.

The second step of the data analysis was to examine the relationship between teacher knowledge and teachers' professional noticing. First, using estimated scores for each component of teacher knowledge and each aspect of teacher noticing as manifest variables, Pearson correlational analyses were conducted between participants' 
knowledge (GPK, MCK, and MPCK) and noticing (P_PID and M_PID). Afterward, with the use of estimated scores for each sub-component of teacher knowledge and each sub-aspect of teacher noticing as manifest variables, Pearson correlational analyses were conducted between each sub-component of participants' knowledge and each sub-aspect of teacher noticing. For MCK, as argued earlier, a higher level of cognitive understanding of mathematics may be more positively related to teacher noticing. Therefore, the focus was changed to its cognitive domains: knowing, applying, and reasoning other than the content domains of mathematics, such as algebra and geometry. Subsequently, path analyses were conducted to examine the joint effect of teacher knowledge (including GPK, MCK, and MPCK) on teacher noticing (including P_PID and M_PID). Mplus was used for the path analyses.

\section{Results}

\section{Correlation Analysis}

Statistically significant but weak positive correlations were identified between the overall constructs GPK and P_PID $(r=0.14)$ and M_PID $(r=0.22)$, as shown in Table 3. In addition, a relatively stronger significant positive correlation was identified between MPCK and M_PID $(r=0.34)$. However, the three correlation coefficient values are all less than 0.4 , indicating a not very strong correlation between them. Moreover, correlations between MCK and P_PID and M_PID as well as the correlation between MPCK and P_PID were rather weak.

The results shown in Table 3 are consistent with findings identified in previous studies with Western teachers. For example, Blömeke et al. (2016) found that GPK is configurationally strongly related to teacher noticing (including P_PID and M_PID). König et al. (2014) also identified a weak but significant correlation between GPK and P_PID. In contrast to the findings in most previous studies in Western culture, no significant correlation between MCK and teacher noticing was identified in the present study with East Asian teachers.

To further examine how each of the sub-domains of mathematics teacher knowledge was related to the sub-facets of their professional noticing, Pearson's $r$ based on the estimated scores of the nine sub-domains of teacher knowledge (GPK, MPCK, and MCK) and the four sub-facets of teacher noticing (P_PID and M PID) was estimated. Table 4 summarizes the results. As illustrated in Table 4 , the relationships between the nine sub-domains of teacher knowledge and the four sub-facets of teacher noticing were all positive, but often quite weak, suggesting that different sub-domains of teacher knowledge are a factor influencing different phases of teacher noticing. However, for Chinese mathematics teachers, it was not in a very strong manner.

Table 3 Correlations between Chinese teacher knowledge and teacher noticing (Pearson's $r ; n=$ 203)

$* p<0.05, * * p<0.01$ $* * * p<0.001$

\begin{tabular}{lll}
\hline & P_PID & M_PID \\
\hline GPK & $0.14^{*}$ & $0.22^{* *}$ \\
MCK & 0.02 & 0.08 \\
MPCK & 0.08 & $0.34^{* * *}$ \\
\hline
\end{tabular}


More specifically, different patterns can be identified from the results in Table 4. Firstly, there are nearly no significant correlations between teacher knowledge and P_PID as a sub-facet of teacher noticing, except for the statistically significant positive correlations between "structure" and "adaptivity" as sub-domains of GPK and "interpretation and decision-making" of P_PID ( $r=0.21$ and 0.16 , respectively). This pattern within the findings is probably influenced by the tendency of the Chinese teachers in P_PID to focus on content-related aspects of teaching, which is evaluated by aspects of M_PID (Yang et al., 2019). The Chinese tradition that most teachers will make their lesson plans highly structured and coherent (Huang \& Li 2009) can explain the unexpected significant correlation between GPK-structure and P_PID, which differs from Western patterns. The weak correlation between "adaptivity" and P_PID also reflects the general tendency of Chinese teachers to adapt their teaching to the whole class as their progress is important in light of the importance of national tests. However, more differentiated views on individual students are less important. In addition, motivation is not that important in Chinese teaching tradition as students are motivated both intrinsically and extrinsically, but most of the time, more extrinsically (Leung, 2001).

Secondly, no significant correlations between perception as the first phase of teacher noticing and all the sub-domains of teacher knowledge can be identified. Such pattern is consistent with findings from our own previous studies comparing Chinese and German teachers' noticing (Yang et al., 2019), in which the Chinese teachers showed their strengths in interpretation and decision-making and showed clear weaknesses in perception. This tendency is probably caused by the fact that most incidents in Chinese classrooms are predictable, such as answers to the teacher's questions. Unexpected incidents which would need strong perception of individual students and their behavior usually do not take place.

Thirdly, there are almost no significant correlations between MCK and all phases of teacher noticing except for a significant correlation between "applying" as a subdomain of MCK and "interpretation and decision-making" of M_PID $(r=0.21)$. These

Table 4 Correlations between sub-domains of Chinese teacher knowledge and sub-facets of teacher noticing (Pearson's $r ; n=203$ )

\begin{tabular}{|c|c|c|c|c|c|}
\hline & & \multicolumn{2}{|l|}{ P_PID } & \multicolumn{2}{|l|}{ M_PID } \\
\hline & & Perception & $\begin{array}{l}\text { Interpretation and } \\
\text { decision-making }\end{array}$ & Perception & $\begin{array}{l}\text { Interpretation and } \\
\text { decision-making }\end{array}$ \\
\hline \multirow[t]{4}{*}{ GPK } & Structure & 0.06 & $0.21 * *$ & 0.08 & $0.17^{* *} *$ \\
\hline & Motivation & 0.07 & 0.13 & 0.07 & 0.11 \\
\hline & Adaptivity & 0.06 & $0.16^{*}$ & 0.03 & 0.12 \\
\hline & Assessment & 0.08 & 0.10 & 0.02 & $0.24 * *$ \\
\hline \multirow[t]{3}{*}{ MCK } & Knowing & 0.01 & 0.03 & 0.09 & 0.13 \\
\hline & Applying & 0.04 & 0.12 & 0.03 & $0.21 * *$ \\
\hline & Reasoning & 0.03 & 0.06 & 0.04 & 0.09 \\
\hline \multirow[t]{2}{*}{ MPCK } & Curriculum & 0.10 & 0.06 & 0.13 & $0.27 * * *$ \\
\hline & Planning and enacting & 0.03 & 0.12 & 0.06 & $0.36^{* * *}$ \\
\hline
\end{tabular}

$* p<0.05, * * p<0.01, * * * p<0.001$ 
results can be explained by the high relevance of practical-algorithmic views on mathematics in Eastern cultures in contrast to that of Western countries, where dialectical-theoretical views on mathematics were dominant (Siu, 2009). This tradition provides interpretations for the missing significant correlations between "knowing" and "reasoning" as sub-domains of MCK and teacher noticing. Overall, this result emphasizes that there are relevant differences in the structure of the knowledge of Chinese teachers, in detail. Mathematical knowledge is used by Chinese teachers under a more instrumental perspective.

Fourthly, the sub-domains of teachers' GPK and MPCK correlate relatively stronger with the sub-facets "interpretation and decision-making" of both P_PID and M_PID. Overall, as reported in Tables 3 and 4, teacher knowledge related to the mathematics instructional (MPCK) or general pedagogical aspects (GPK) tended to be more closely related to teacher noticing, especially for the sub-domain "interpretation and decisionmaking," which is in line with our previous results comparing German and Chinese teachers' noticing (Yang et al., 2019). The correlation between Chinese mathematics teachers' MCK (including their cognitive sub-domains) and their noticing, even on the M_PID sub-facet, was not significant, which suggests that different sub-domains of teacher knowledge function in differentiated manners at different parts of teacher noticing in an East Asian context compared with a Western context.

\section{Path Analysis}

A path analysis was conducted to examine the joint relationships between Chinese mathematics teachers' knowledge, including GPK, MCK, and MPCK, on the one hand, and their professional noticing, including P_PID and M_PID, on the other hand. The path model fits the data well, $\chi^{2} / \mathrm{df}=2.15, \bar{p}<0.001 ; \overline{\mathrm{CFI}}=0.929 ; \mathrm{RMSEA}=0.050$; $\mathrm{SRMR}=0.052$. Figure 2 represents the results of the global effect of GPK, MCK, and MPCK on teacher noticing, including P_PID and M_PID.

First, the knowledge domains GPK, $\bar{M}$ CK, and MPCK did not significantly predict teacher noticing on the aspect of P_PID. As shown in Fig. 2, the path coefficient between MCK and P_PID was rather weak $(\beta=0.02)$, which was expected. However, the path coefficient between GPK and P_PID was relatively stronger than the other two $(\beta=0.11)$, but was in contrast to the Pearson's correlation reported above, which was

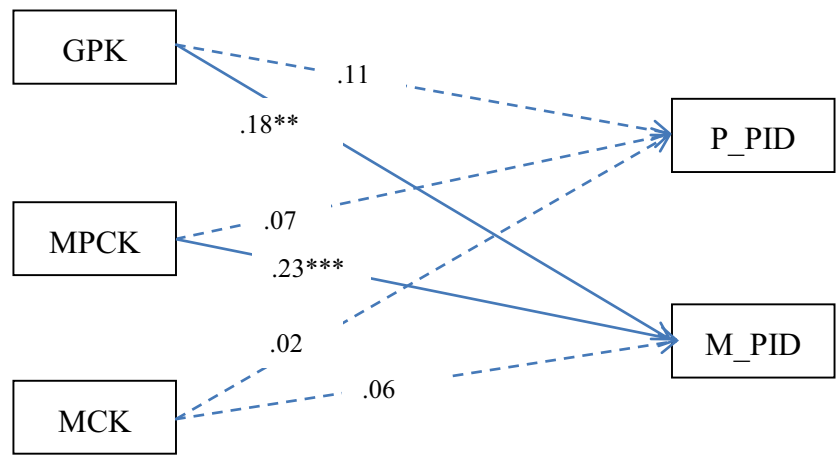

Fig. 2 Path analysis results for the relationship among teacher knowledge and teacher noticing. The dotted lines represent non-significant path coefficients. 
not significant. This result does not support our second hypothesis. The relation between MPCK and P_PID was not significant either $(\beta=0.07)$.

Second, both GPK and MPCK positively and significantly predicted teachers' noticing on the aspect of M_PID. As shown in Fig. 2, the path coefficients between GPK and MPCK and M_PID were the two largest among all the six path coefficients. However, the relations between teachers' MCK and their M_PID were again found to be not significant $(\beta=0.06)$. The associations between teachers' GPK, MCK, and MPCK and teachers' P_PID and M_PID may again imply that teachers' pedagogy-related knowledge, including GPK and MPCK, has a more powerful influence on teachers' mathematics instruction-related noticing, which is in line with our first hypothesis. MCK does not have a direct influence on teachers' noticing in general and mathematics instruction-related noticing in particular. Although we hypothesized a weaker relationship between MCK and teacher noticing, it is an unexpected result that no systematic relationship existed at all.

\section{Discussion}

This study aimed to investigate the relationship between Chinese mathematics teachers' GPK, MCK, and MPCK and their noticing competence from a mathematics instructional and pedagogical perspective. As reported, the relationship between Chinese teachers' knowledge and their professional noticing was weak in contrast to the empirically identified strong relation in Western culture as expected in our hypotheses due to the culturally shaped nature of knowledge-based facets of teachers' competence and situated facets of teachers' competence. This applied to the relationships between the overall constructs as well as to the relationships between the nine sub-domains of teacher knowledge and the four sub-facets of teacher noticing, which were mostly not significant. In particular, the fact that the correlation between Chinese mathematics teachers' MCK (including sub-domains) and their noticing was not significant at all, even on the M_PID sub-facet, was expected and in line with our first hypothesis, as we had hypothesized a weaker relationship of Chinese teachers' MPCK and GPK to noticing compared with Western teachers. This hypothesis was supported by the data, especially with respect to the sub-domain "interpretation and decision-making." Furthermore, the mediating role of MPCK was supported by the data from GPK to M_PID.

First, the correlation and path analysis results in the study suggest that different components of teacher knowledge correlate to teacher noticing differently. Compared with the association between teachers' pedagogy-related knowledge (such as GPK and MPCK) and their noticing, the association between teachers' MCK and their noticing is weak and independent from the level of cognitive understanding of mathematics. Partially similar findings were identified in a few studies conducted in Western contexts (e.g. Dreher \& Kuntze, 2015). Blömeke et al. (2016) also found that teachers' GPK was structurally more closely grouped related to teacher noticing, not their MCK and MPCK. Therefore, findings in the study indeed confirm that teacher noticing, content knowledge, pedagogical content knowledge, and general pedagogical knowledge are not of "the same nature" (Blömeke et al., 2016, p. 44).

Second, it is noteworthy to point out that for a specific type of teacher knowledge (e.g. GPK and MPCK), Chinese mathematics teachers' knowledge tended to be more closely correlated with the "interpretation and decision-making" facets of teachers' noticing than 
with the "perception" facet. In particular, the correlation analysis results between the subdomains of teacher knowledge and different phases of teacher noticing clearly show that no significant correlation existed between each sub-domain of teacher knowledge and the perception facet of teacher noticing. This result is in part also consistent with previous findings in Western culture. For example, König et al. (2014) identified a strong association between teachers' GPK and their skills to interpret $(\beta=0.45)$, but a quite weak association between teachers' GPK and their skills to perceive $(\beta=0.07)$. Similarly, recently, Sánchez-Matamoros et al. (2019) found that only pre-service teachers' ability to interpret and decide are strongly connected to their content knowledge and knowledge of students' mathematical thinking, and not the ability to attend to incidents.

This tendency might be due to the theoretical differences between the constructs "perception" and "interpretation and decision-making" (Kaiser et al., 2015; Sherin \& Star, 2011). In addition, in a complex classroom environment, teachers' choice to attend or ignore an event is a more intuitive action (Sherin \& Star, 2011). It might be more highly influenced by teachers' orientations, especially by their prior relevant experience (Schoenfeld, 2011). However, the latter two processes of teachers' professional noticing, namely, interpretation and decision-making, are more knowledge-based reasoning behaviors (Sherin et al., 2011; van Es, Cashen, Barnhart, \& Auger, 2017). Therefore, it is possible that no matter which part of teacher knowledge is focused on, it tends to be more closely related to the "interpretation" and "decision-making" processes. This may further imply that teachers' knowledge functions differently in different processes of teachers' noticing. Overall, more studies are needed in this field before secure conclusions can be made.

Finally, consistent with our main hypothesis, the results of our study point out that the overall correlation between teacher knowledge and teacher noticing for Chinese inservice mathematics teachers is much weaker than the correlations identified in Western culture. The available results in the existing studies from Western culture tend to show that the two constructs are "moderately related" (Meschede, Fiebranz, Möller, \& Steffensky, 2017, p. 167). In the present study, only quite few of the coefficients for correlation analysis and path analysis show a significant correlation at moderate level. In particular, for the relationship between MCK and teacher noticing, no significant correlation was identified in the present study, not even for any sub-facets of M_PID. These results are, as discussed above, in contrast to results from studies in Western culture, which found moderate or high correlation between MCK and teacher noticing (Bruckmaier et al., 2016; Kersting et al., 2012; König et al., 2014).

However, differences as such do not suggest that for Chinese teachers, teacher knowledge is not important or needed for successful teacher noticing. On the contrary, it may first echo that declarative knowledge only is not sufficient for teacher noticing, but other factors such as teacher orientations (Schoenfeld, 2011) or practical experience (Meschede et al., 2017) may jointly play a decisive role. Teacher orientations and practical experience, however, are developed or accumulated within a specific context, and therefore, heavily influenced by traditions and culture. Therefore, the weak or insignificant associations between Chinese in-service mathematics teachers' knowledge and their noticing may be very much caused by specific mathematics education tradition and culture. Some characteristics have already been mentioned above. In the following, we will elaborate on these differences more extensively and intensively.

Firstly, Chinese (mathematics) teaching and working culture and tradition can provide explanations to the weak associations between teacher knowledge and teacher 
noticing. It was found in comparative studies that unlike Western (e.g. US) teachers, Chinese teachers pre-design their teaching carefully by studying textbooks and studying possible student thinking processes beforehand thoroughly (Cai, Ding \& Wang, 2014; Fang \& Gopinathan, 2009). The intensive study of textbooks and students' dispositions, like students' prior knowledge and learning experience, will help teachers "precisely predict the possible difficult concepts for their students" (Cai \& Wang, 2010, p. 278). With the understanding or estimating of students' possible difficulties, Chinese teachers will not only design the teaching sequences and transitional language, but also a variation of tasks or exercises and even questions beforehand (Cai et al., 2014; Huang et al., 2011). In other words, Chinese teachers have the tradition to study students before teaching and make themselves well prepared instead of trying to understand students during or after teaching like teachers in Western culture usually do (Cai \& Wang, 2010). Such a tradition of teaching and working culture may lead the Chinese teachers to pay more attention to their well-designed teaching script but less attention to those aspects that they did not predict beforehand.

In addition, it is also well known that the class size in China is normally larger than 50 students. Therefore, mathematics teaching usually takes place in a large group setting. The most popular mathematics teaching model traditionally in China is direct teaching to the whole class (Leung, 2001). Although under the influence of the latest mathematics curriculum reform started in 2001 which encouraged student-centered teaching, empirical studies on mathematics teaching practice still found that mathematics teaching in China is characterized as "teacher-centered questions and answers" (Li $\& \mathrm{Ni}, 2011$, p. 83). Substantial student participation is rare and demonstration of student-centered features is tightly teacher-controlled (Mok, 2006). Overall, direct lecturing and explaining still dominates in whole classroom instructions (Huang \& Li, 2009). Such a direct model of teaching was described as the "virtuoso model" by Western researchers (Paine, 1990). In this model, the role of teachers is mainly to lead or guide the whole class by perfectly demonstrating knowledge to students in a highly structured way, and the students are expected to follow and concentrate on their teachers' teaching (Fang \& Gopinathan, 2009). Therefore, this direct teaching tradition may also weaken the association between teacher knowledge and teacher noticing.

Moreover, due to the high pressure from the dominant examination culture in China, another tradition for Chinese teachers is that they are always supposed to complete a certain amount of content within each lesson. Therefore, each mathematics lesson should have a definite and clear objective, which usually focuses on the learning of basic mathematical knowledge and the development of basic mathematical skills (Zheng, 2006). Teachers are also supposed to highlight the essential points (重点) of the teaching content and help students solve difficult points (难点) by appropriate instructions, which support students in reaching profound understanding. In terms of mathematical understanding, Chinese mathematics teachers were found to emphasize more the process of mathematical concept development and students' grasping the essence of a concept as the core of understanding (Cai \& Ding, 2017). Correspondingly, in teaching, Chinese teachers tend to pay more attention to internal mathematics connections with emphasis on mathematical reasoning, knowledge construction, and development (Huang \& Li, 2009). Based on such tradition of emphasizing the mastery or understanding of mathematics content, in practice, Chinese mathematics teachers will care more about students' mental engagement and ignore other aspects of things 
happening in their teaching (Ding, Li, Li, \& Kulm, 2008). Indeed, in line with these results concerning the noticing perspective, Chinese mathematics teachers also tend to focus on content-related aspects of teaching, namely, the M_PID-related aspects (Yang et al., 2019). Overall, this Chinese's mathematics teaching culture may weaken the correlation between Chinese mathematics teachers' knowledge and their noticing.

Secondly, the tradition of teacher education in China may act as another influential factor for the weak association. Pre-service teacher education in China has a tradition of emphasizing strongly the learning of academic knowledge. In China, more than $80 \%$ of the courses during pre-service teacher education are subject-related theoretical courses (e.g. advanced mathematics courses like analysis and advanced algebra), with limited attention to pedagogy and teaching skills practice (Paine, Fang, \& Wilson, 2003). This tradition of curriculum policy is characteristic for Chinese teacher education, but is criticized in Western countries, describing teacher education as a low-impact enterprise (Lortie, 1975) or with the double discontinuity hypothesis developed by Felix Klein more than 100 years ago. According to Klein (1904), school mathematics and university mathematics are disconnected areas; as the knowledge facets belong to the university mathematics and noticing facets refer to school mathematics, it can be expected that in the course of teachers' professional development, this relation will develop at a different level. However, comparing Chinese and at least German teachers' education, this gap between the relevance of highly demanding mathematical courses with strong emphasis on subject-knowledge and pedagogical courses is much stronger in China than in Germany, which has a typical Western teacher education, according to the TEDS-M study (Tatto et al., 2008).

Thirdly, the school-based professional development culture can provide another explanation. Like other Eastern countries, under the influence of collectivism, teachers in China share a long tradition of planning together and observing each other's lessons (Fang \& Gopinathan, 2009). In fact, in China, newly graduated teachers are never regarded as qualified teachers, but as "semi-finished products" (Paine et al., 2003, p. 216) who need to learn and develop their teaching skills after they enter teaching positions in schools through mentoring by experienced teachers and observing other teachers' classes. Knowledge learned and experience accumulated by such means, however, are mainly implicit and can be used without awareness and verbal explication (Elliott, Stemler, Sternberg, Grigorenko, \& Hoffman, 2011; König et al., 2014). In practice, such tacit knowledge and experience may help teachers easily notice relevant incidents in a complex classroom environment. However, it is a challenging task to measure it since it is "more sensitive to the context in which the behavior occurs" (Taub, Hayes, Cunningham, \& Sivo, 2001, p. 941). Overall, this kind of experience by Chinese teachers may also be an influential factor for the weak correlation between teacher knowledge and teacher noticing.

\section{Conclusions and Limitations}

Although it has been theoretically assumed and empirically found that teacher knowledge has a fundamental impact on teachers' noticing in Western culture, limited empirical evidence is available thus far to support such argument for other cultural contexts. Our study aimed to close this gap with standardized testing instruments. Our study points out that for Chinese teachers, the components of teacher knowledge (e.g. MCK, MPCK, and GPK) are of varying influence on teacher noticing, yielding 
structural patterns different from patterns found in Western contexts so far. Furthermore, East Asian and Western teachers' structural differences between the different subdomains of teacher knowledge and the various phases of teacher noticing can be identified. The differences in the strength of the relations between teacher knowledge and noticing between East Asian and Western teachers and the differences in their structure may be due to social and cultural influences, as described in the "Discussion" part, in which we developed possible explanations for the weaker relations for knowledge and noticing of Chinese teachers compared with their Western counterparts.

Although the present study is one of the few studies thus far to explore mathematics teacher knowledge, including GPK, MCK, and MPCK, and teachers' noticing with Chinese teachers, the limitations of the study should also be discussed. First, participants in the study were mainly chosen from one major city/region in China and only from junior secondary school level. Therefore, to a certain degree, the sample in the present study may not be typical enough to reflect the general situation or diversity of mathematics teachers' professional noticing in China. Moreover, another weakness of the present study is that it assessed teachers' knowledge and teachers' noticing in a more general way. That is, the present study did not focus on the relationship between teachers' knowledge and teachers' noticing on a specific branch of mathematics (e.g. geometry or algebra) or on a specific mathematical topic (e.g. fractions or equations).

In addition, considering that every participant in the present study needed to spend two and a half hours to complete the five assessments, shorter versions of the original knowledge instruments were employed in the present study to lessen the test burden for participants. Therefore, although the reliabilities for most of the five assessment instruments were acceptable, it is possible that each component of teachers' knowledge and teachers' noticing was not fully assessed. Further studies should consider employing relatively longer instruments to assess all the sub-domains of GPK, MCK, and MPCK more comprehensively with teachers for other age cohorts in order to enhance the validity and reliability of the instruments and broaden the results.

Finally, an overall picture on the complex relation between teachers' knowledge and teachers' noticing may need to include additional other factors such as teacher beliefs and teaching experiences as teachers' competences are of complex nature, shaped by the cultural context.

Acknowledgments Exploring the Eastern and the Western Debate: the Case of Mathematics Teachers' Professional Knowledge and Students' Achievement - A Comparative Study between Germany and China, EU-financed in the frame of the Marie Sklodowska-Curie grant, grant number: 658303, project duration: 2016-2018.

Funding Information Open access funding provided by Projekt DEAL.

Open Access This article is licensed under a Creative Commons Attribution 4.0 International License, which permits use, sharing, adaptation, distribution and reproduction in any medium or format, as long as you give appropriate credit to the original author(s) and the source, provide a link to the Creative Commons licence, and indicate if changes were made. The images or other third party material in this article are included in the article's Creative Commons licence, unless indicated otherwise in a credit line to the material. If material is not included in the article's Creative Commons licence and your intended use is not permitted by statutory regulation or exceeds the permitted use, you will need to obtain permission directly from the copyright holder. To view a copy of this licence, visit http://creativecommons.org/licenses/by/4.0/. 


\section{References}

Ball, D. L. (2011). Foreword. In M. G. Sherin, V. R. Jacobs, \& R. A. Philipp (Eds.), Mathematics teacher noticing: Seeing through teachers' eyes (pp. xx-xxiv). New York: Routledge.

Ball, D. L., Thames, M. H., \& Phelps, G. (2008). Content knowledge for teaching: What makes it special? Journal of Teacher Education, 59, 389-407.

Bentler, P. M., \& Chou, C. P. (1987). Practical issues in structural modeling. Sociological Methods \& Research, 16, 78-117.

Berliner, D. C. (2001). Learning about and learning from expert teachers. International Journal of Educational Research, 35(5), 463-482.

Beswick, K., Callingham, R., \& Watson, J. (2012). The nature and development of middle school mathematics teachers' knowledge. Journal of Mathematics Teacher Education, 15(2), 131-157.

Blömeke, S., Busse, A., Kaiser, G., König, J., \& Suhl, U. (2016). The relation between content-specific and general teacher knowledge and skills. Teaching and Teacher Education, 56, 35-46.

Blömeke, S., Suhl, U., \& Döhrmann, M. (2013). Assessing strengths and weaknesses of teacher knowledge in Asia, Eastern Europe and Western countries: Differential item functioning in TEDS-M. International Journal of Science and Mathematics Education, 11, 795-817.

Bond, T., \& Fox, C. (2007). Applying the Rasch model: Fundamental measurement in the human sciences (2nd ed.). Mahwah: LEA.

Bruckmaier, G., Krauss, S., Blum, W., \& Leiss, D. (2016). Measuring mathematics teachers' professional competence by using video clips (COACTIV video). ZDM Mathematics Education, 48(1-2), 111-124.

Cai, J., \& Ding, M. (2017). On mathematical understanding: Perspectives of experienced Chinese mathematics teachers. Journal of Mathematics Teacher Education, 20(1), 5-29.

Cai, J., Ding, M., \& Wang, T. (2014). How do exemplary Chinese and US mathematics teachers view instructional coherence? Educational Studies in Mathematics, 85(2), 265-280.

Cai, J., \& Wang, T. (2010). Conceptions of effective mathematics teaching within a cultural context: Perspectives of teachers from China and the United States. Journal of Mathematics Teacher Education, 13(3), 265-287.

Ding, M., Li, Y., Li, X., \& Kulm, G. (2008). Chinese teachers' perceptions of students' classroom misbehaviour. Educational Psychology, 28(3), 305-324.

Dreher, A., \& Kuntze, S. (2015). Teachers' professional knowledge and noticing: The case of multiple representations in the mathematics classroom. Educational Studies in Mathematics, 88(1), 89-114.

Dunekacke, S., Jenßen, L., Eilerts, K., \& Blömeke, S. (2016). Epistemological beliefs of prospective preschool teachers and their relation to knowledge, perception, and planning abilities in the field of mathematics: A process model. ZDM Mathematics Education, 48(1), 125-137.

Elliott, J. G., Stemler, S. E., Sternberg, R. J., Grigorenko, E. L., \& Hoffman, N. (2011). The socially skilled teacher and the development of tacit knowledge. British Educational Research Journal, 37(1), 83-103.

Fang, Y., \& Gopinathan, S. (2009). Teachers and teaching in eastern and western schools: A critical review of cross-cultural comparative studies. In L. J. Saha \& A. G. Dworkin (Eds.), International handbook of research on teachers and teaching (pp. 557-572). New York: Springer.

Fennema, E., \& Franke, M. L. (1992). Teachers' knowledge and its impact. In D. A. Grouws (Ed.), Handbook of mathematics teaching and learning (pp. 147-164). New York: Macmillan.

Hambleton, R. K. (2005). Issues, designs, and technical guidelines for adapting tests into multiple languages and cultures. In R. K. Hambleton, P. F. Merenda, \& C. D. Spielberger (Eds.), Adapting educational and psychological tests for crosscultural assessment (pp. 3-38). Mahwah: Erlbaum.

Hill, H. C., Dean, C., \& Goffney, I. M. (2007). Assessing elemental and structural validity: Data from teachers, non-teachers, and mathematicians. Measurement, 5(2-3), 81-92.

Huang, R. \& Li, Y. (2009). Pursuing excellence in mathematics classroom instruction through exemplary lesson development in China: A case study. ZDM Mathematics Education, 41, 297-309.

Huang, R., Li, Y., Zhang, J., \& Li, X. (2011). Improving teachers' expertise in mathematics instruction through exemplary lesson development. ZDM Mathematics Education, 43(6-7), 805-817.

Jacobs, V. R., Lamb, L. L. C., \& Philipp, R. A. (2010). Professional noticing of children's mathematical thinking. Journal for Research in Mathematics Education, 41, 169-202.

Kaiser, G., \& Blömeke, S. (2013). Learning from the Eastern and the Western debate: The case of mathematics teacher education. ZDM Mathematics Education, 45(1), 7-19.

Kaiser, G., Blömeke, S., König, J., Busse, A., Döhrmann, M., \& Hoth, J. (2017). Professional competencies of (prospective) mathematics teachers-Cognitive versus situated approaches. Educational Studies in Mathematics, 94(2), 161-182. 
Kaiser, G., Busse, A., Hoth, J., König, J., \& Blömeke, S. (2015). About the complexities of vid-eo-based assessments: Theoretical and methodological approaches to overcoming shortcomings of research on teachers' competence. International Journal of Science and Mathematics Education, 13(2), 369-387.

Kane, M. T. (2001). Current concerns in validity theory. Journal of Educational Measurement, 38(4), 319342.

Kersting, N. B., Givvin, K. B., Thompson, B. J., Santagata, R., \& Stigler, J. W. (2012). Measuring usable knowledge: Teachers' analyses of mathematics classroom videos predict teaching quality and student learning. American Educational Research Journal, 49(3), 568-589.

Kleickmann, T., Richter, D., Kunter, M., Elsner, J., Besser, M., Krauss, S., Cheo, M., \& Baumert, J. (2015) Content knowledge and pedagogical content knowledge in Taiwanese and German mathematics teachers. Teaching and Teacher Education, 45 ,115-126.

Klein, F. (1904). Elementarmathematik vom höheren Standpunkte aus. Vol. 1. Leipzig: Springer. Newly translated (2016), Elementary mathematics from a higher standpoint. Cham: Springer.

König, J., Blömeke, S., Klein, P., Suhl, U., Busse, A., \& Kaiser, G. (2014). Is teachers' general pedagogical knowledge a premise for noticing and interpreting classroom situations? A video-based assessment approach. Teaching and Teacher Education, 38, 76-88.

König, J., Blömeke, S., Paine, L., Schmidt, W. H., \& Hsieh, F.-J. (2011). General pedagogical knowledge of future middle school teachers: On the complex ecology of teacher education in the United States, Germany, and Taiwan. Journal of Teacher Education, 62, 188-201.

Leung, F. K. S. (2001). In search of an East Asian identity in mathematics education. Educational Studies in Mathematics, 47(1), 35-51.

Li, Q., \& Ni, Y. (2011). Impact of curriculum reform: Evidence of change in classroom practice in mainland China. International Journal of Educational Research, 50(2), 71-86.

Little, T. D., Cunningham, W. A., Shahar, G., \& Widaman, K. F. (2002). To parcel or not to parcel: Exploring the question weighing the merits. Structural Equation Modeling, 9, 151-173.

Lortie, D. (1975). Schoolteacher: A sociological study. London: University of Chicago Press.

Louie, N. L. (2018). Culture and ideology in mathematics teacher noticing. Educational Studies in Mathematics, 97(1), 55-69.

Mason, J. (1998). Enabling teachers to be Real teachers: Necessary levels of awareness and structure of attention. Journal of Mathematics Teacher Education, 1, 243-267.

Mason, J. (2009). Teaching as disciplined enquiry. Teacher and Teaching: theory and practice, 15(2), 205223.

Meschede, N., Fiebranz, A., Möller, K., \& Steffensky, M. (2017). Teachers' professional vision, pedagogical content knowledge and beliefs: On its relation and differences between pre-service and in-service teachers. Teaching and Teacher Education, 66, 158-170.

Mok, I. (2006). Teacher-dominating lesson in Shanghai: The insiders' story. In D. J. Clarke, C. Keitel, \& Y. Shimizu (Eds.), Mathematics classrooms in twelve countries: The insider' perspective (pp. 87-98). Rotterdam: Sense.

Paine, L. W. (1990). The teacher as virtuoso: A Chinese model for teaching. Teachers College Record, 92(1), 49-81.

Paine, L. W., Fang, Y., \& Wilson, S. (2003). Entering a culture of teaching. In E. Britton, L. Paine, D. Pimm, \& S. Raizen (Eds.), Comprehensive teacher induction: Systems for early career learning (pp. 20-82). Dordrecht: Kluwer.

Sánchez-Matamoros, G., Fernández, C., \& Llinares, S. (2019). Relationships among prospective secondary mathematics teachers' skills of attending, interpreting and responding to students' understanding. Educational Studies in Mathematics, 100(1), 83-99.

Schoenfeld, A. H. (2011). Noticing matters. A lot. Now what? In M. G. Sherin, V. R. Jacobs, \& R. A. Philipp (Eds.), Mathematics teacher noticing: Seeing through teachers' eyes (pp. 223-238). New York: Routledge.

Sherin, B., \& Star, J. R. (2011). Reflections on the study of teacher noticing. In M. G. Sherin, V. R. Jacobs, \& R. A. Philipp (Eds.), Mathematics teacher noticing: Seeing through teachers' eyes (pp. 66-78). New York: Routledge.

Sherin, M. G., Jacobs, V. R., \& Philipp, R. A. (Eds.). (2011). Mathematics teacher noticing: Seeing through teachers' eyes. New York: Routledge.

Sherin, M. G., \& van Es, E. A. (2005). Using video to support teachers' ability to notice classroom interactions. Journal of Technology and Teacher Education, 13, 475-491.

Sherin, M. G., \& van Es, E. A. (2009). Effects of video club participation on teachers' professional vision. Journal of Teacher Education, 60, 20-37. 
Shulman, L. (1987). Knowledge and teaching: Foundations of the new reform. Harvard Educational Review, $57,1-22$.

Siu, M. K. (2009). Mathematics education in East Asia from antiquity to modern times. In K. Bjarnadottir, F. Furinghetti, \& G. Schubring (Eds.), Dig where you stand: Proceedings of the Conference on On-going Research in the History of Mathematics Education, Gardabaer (pp. 197-208). Reykjav1'k: School of Education of University of Iceland.

Stockero, S. L., Ropnow, R. L., \& Pascoe, A. E. (2017). Learning to notice important student mathematical thinking in complex classroom interactions. Teaching and Teacher Education, 63, 384-395.

Stürmer, K., Könings, K. D., \& Seidel, T. (2013). Declarative knowledge and professional vision in teacher education: Effect of courses in teaching and learning. British Journal of Educational Psychology, 83(3), $467-483$.

Tatto, M. T., Schwille, J., Senk, S. L., Ingvarson, L., Peck, R., \& Rowley, G. (2008). Teacher Education and Development Study in Mathematics (TEDS-M): Policy, practice, and readiness to teach primary and secondary mathematics. East Lansing: Teacher Education and Development International Study Center, Michigan State University.

Taub, G. E., Hayes, B. G., Cunningham, W. R., \& Sivo, S. A. (2001). Relative roles of cognitive ability and practical intelligence in the prediction of success. Psychological Reports, 88, 931-942.

Thomas, J. N. (2017). The ascendance of noticing: Connections, challenges, and questions. In E. O. Schack, H. F. Molly, \& J. A. Wilhelm (Eds.), Teacher noticing: Bridging and broadening perspectives, contexts, and frameworks (pp. 507-514). New York: Springer.

van Es, E. A., Cashen, M., Barnhart, T., \& Auger, A. (2017). Learning to notice mathematics instruction: Using video to develop preservice teachers' vision of ambitious pedagogy. Cognition and Instruction, 35(3), 1-23.

Van Es, E. A., \& Sherin, M. G. (2002). Learning to notice: Scaffolding new teachers' interpretations of classroom interactions. Journal of Technology and Teacher Education, 10(4), 571-596.

Yang, X., Kaiser, G., König, J., \& Blömeke, S. (2018). Measuring Chinese teacher professional competence: Adapting and validating a German framework in China. Journal of Curriculum Studies, 50(5), 638-653.

Yang, X., Kaiser, G., König, J., \& Blömeke, S. (2019). Professional noticing of mathematics teachers: A comparative study between Germany and China. International Journal of Science and Mathematics Education, 17, 943-963.

Zheng, Y. (2006). Mathematics education in China: From a cultural perspective. In F. K. S. Leung, K. D. Graf, \& F. Lopez-Real (Eds.), Mathematics education in different cultural traditions-A comparative study of East Asia and the West (pp. 381-390). New York: Springer.

\section{Affiliations}

\section{Xinrong Yang ${ }^{1} \cdot$ Gabriele Kaiser $^{2} \cdot$ Johannes König $^{3} \cdot$ Sigrid Blömeke $^{4}$}

1 Southwest University, No. 2 Tiansheng Road, Beibei District, Chongqing 400715, China

2 Universität Hamburg/Australian Catholic University, Von-Melle-Park 8, 20146 Hamburg, Germany

3 University of Cologne, Gronewaldstraße 2a, 50931 Cologne, Germany

4 University of Oslo/Centre for Educational Measurement, Postboks 1161, Blindern, 0318 Oslo, Norway 